\title{
Glass Transition in a Two-Dimensional Electron System in Silicon in a Parallel Magnetic Field
}

\author{
J. Jaroszyński, ${ }^{1, \text { * }}$ Dragana Popović, ${ }^{1}$ and T. M. Klapwijk ${ }^{2}$ \\ ${ }^{1}$ National High Magnetic Field Laboratory, Florida State University, Tallahassee, Florida 32310 \\ ${ }^{2}$ Department of Applied Physics, Delft University of Technology, 2628 CJ Delft, The Netherlands
}

(Dated: October 28, 2018)

\begin{abstract}
Studies of low-frequency resistance noise show that the glassy freezing of the two-dimensional electron system (2DES) in $\mathrm{Si}$ in the vicinity of the metal-insulator transition (MIT) persists in parallel magnetic fields $B$ of up to $9 \mathrm{~T}$. At low $B$, both the glass transition density $n_{g}$ and $n_{c}$, the critical density for the MIT, increase with $B$ such that the width of the metallic glass phase $\left(n_{c}<n_{s}<n_{g}\right)$ increases with $B$. At higher $B$, where the 2DES is spin polarized, $n_{c}$ and $n_{g}$ no longer depend on $B$. Our results demonstrate that charge, as opposed to spin, degrees of freedom are responsible for glassy ordering of the 2DES near the MIT.

PACS numbers: 71.30.+h, 71.27.+a, 73.40.Qv
\end{abstract}

The fascinating strong correlation physics exhibited by low-density two-dimensional (2D) electron and hole systems [1] remains the subject of intensive research. In the vicinity of the apparent metal-insulator transition (MIT), in particular, both electron-electron interactions and disorder appear to be equally important. Their competition may lead to the emergence of many metastable states and the resulting glassy dynamics of electrons. Recent experiments 2, 3] on a $2 \mathrm{D}$ electron system in Si have demonstrated such glassy behavior, lending support to the theoretical proposals that attempt to describe the 2D MIT as the melting of a Coulomb [4, 5], Wigner [6], or spin 7 glass. Even though several features of the data 2, 3] are consistent with the model of glassy behavior that occurs in the charge sector [5, 8, 9], it is still an open question whether charge or spin degrees of freedom are responsible for the observed glass transition. Since a sufficiently strong magnetic field is expected to destroy the spin glass order 7, 10], experimental studies of glassy dynamics in parallel magnetic fields $B$ [1] should be able to distinguish between the proposed models. Here we present such a study, which shows that the glass transition persists even in $B$ such that the $2 \mathrm{D}$ system is spin polarized. These results demonstrate that charge, as opposed to spin, degrees of freedom are responsible for glassy ordering of the 2DES near the MIT.

Previous studies carried out at $B=0$ employed a combination of transport and low-frequency resistance noise measurements 2, 3] to probe the glassy behavior. The glass transition was manifested by a sudden and dramatic slowing down of the electron dynamics and by an abrupt change to the sort of statistics characteristic of complicated multistate systems, consistent with the hierarchical picture of glassy dynamics. It was also found [2] that the glass transition occurs in the metallic phase, i.e. at an electron density $n_{g}>n_{c}$, where $n_{c}$ is the critical density for the MIT determined from the vanishing of activation energy in the insulating regime [12, 13]. The intermediate metallic glass (MG) phase is of considerable width in strongly disordered samples $\left(\left(n_{g}-n_{c}\right) / n_{c} \approx 0.5\right.$ [2]), whereas in devices with low disorder $n_{g}$ is only a few percent higher than $n_{c}[\underline{3}$ ], in agreement with theory [9]. In this work, we investigate the glass transition in these same high peak mobility (low disorder) devices using noise spectroscopy in a parallel $B$. We find that all the signatures of the glass transition in parallel $B$ are qualitatively the same as in $B=0$, even when the electrons are spin polarized at high $B$. We construct a phase diagram in the $\left(n_{s}, B\right)$ plane ( $n_{s}$ is the electron density), and find that the MG phase is broadened by a parallel $B$. The temperature $(T)$ dependence of the conductivity $\sigma$ in the MG at low $B$ is similar to the one first observed in the MG phase of highly disordered devices at $B=0$ [2], and it is consistent with theory [8]. We show that, at low $B$, it also provides evidence for a quantum phase transition $(\mathrm{QPT})$ at $n_{c}(B)$.

The experiment was performed on n-channel Si metaloxide-semiconductor field-effect transistors (MOSFETs) with the peak mobility $\mu \approx 2.5 \mathrm{~m}^{2} / \mathrm{Vs}$ at $4.2 \mathrm{~K}$, fabricated in a Hall bar geometry with $\mathrm{Al}$ gates, and oxide thickness $d_{o x}=147 \mathrm{~nm}$ [14]. The resistance $R$ was measured using a standard four-probe ac technique (typically $2.7 \mathrm{~Hz}$ ) in the Ohmic regime. A precision DC voltage standard (EDC MV116J) was used to apply the gate voltage, which controls $n_{s}$. We note that $n_{s}$ was always varied at a relatively high $T \approx 2 \mathrm{~K}$. Contact resistances and their influence on noise measurements were minimized by using a split-gate geometry, which allows one to maintain high $n_{s}\left(\approx 10^{12} \mathrm{~cm}^{-2}\right)$ in the contact region while allowing an independent control of $n_{s}$ of the $2 \mathrm{D}$ system under investigation in the central part of the sample $\left(120 \times 50 \mu \mathrm{m}^{2}\right)$. The samples and the measurement technique have been described in more detail elsewhere [3].

For each $n_{s}$ and $B, R$ was measured as a function of time $t$ at $T=0.24 \mathrm{~K}$, although measurements at higher $T$ were also performed at several selected $B$. At $B=0$, the temperature coefficient of the time-averaged resistivity $d\langle\rho\rangle / d T=0$ at $n_{s}^{*} \approx 9.7 \times 10^{10} \mathrm{~cm}^{-2}$, similar to what 

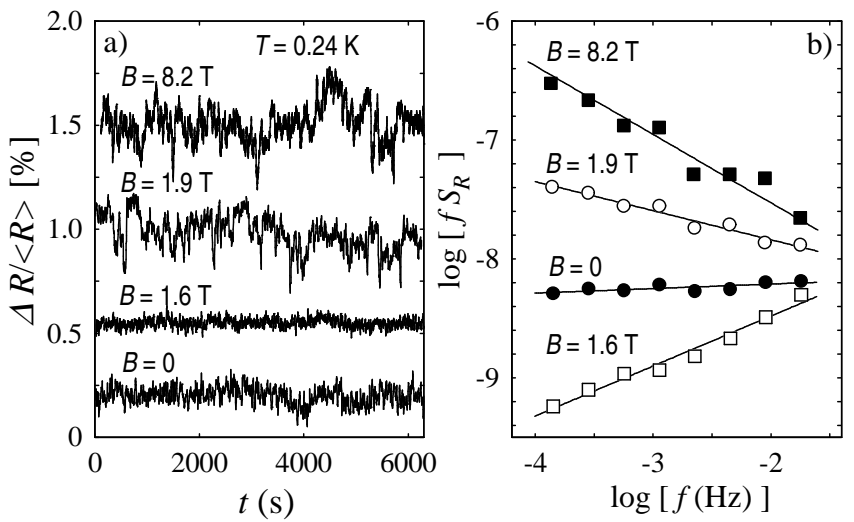

FIG. 1: (a) $\Delta R(t) /\langle R\rangle$, and (b) the corresponding power spectra $S_{R}(f)$, at several $B$ for $n_{s}=11.9 \times 10^{10} \mathrm{~cm}^{-2}$. In (a) traces are shifted for clarity. In (b) $S_{R}(f)$ are averaged over octaves and multiplied by $f$, so that $1 / f$ spectrum is horizontal on this scale. Solid lines are linear least-squares fits with the slopes $\alpha=1.58,1.24,0.96,0.58$ (from top).

was obtained on the previous cooldown of the same sample [3]. At a fixed $T$ and in the range of $n_{s}$ under investigation, $\langle\rho\rangle$ exhibits a dramatic increase with $B$, followed by a much weaker dependence ("saturation") at higher fields $(B>2-4 \mathrm{~T})$. This large positive magnetoresistance at low $B$ has been observed and studied extensively in many 2 D systems [1], including other samples from the same source 14] as ours. In the saturation region, it has been shown 15] that the 2DES is spin polarized.

Figures 1a) and 1(b) show the time series of the relative changes in resistance $\Delta R(t) /\langle R\rangle$ and the corresponding power spectra $S_{R}(f)$, respectively, for a fixed $n_{s}$ and several $B$. It is obvious that $B$ has a strong effect on both the amplitude and the character of the noise, as discussed in detail below. In order to compare the noise magnitudes under different conditions, the power $S_{R}(f=1 \mathrm{mHz})$ is taken as the measure of noise. It is determined from the fits of the octave-averaged spectra to the form $1 / f^{\alpha}$ for $10^{-4}<f<0.07 \mathrm{~Hz}$ [solid lines in Fig. 1(b)]. In addition, we have also analyzed the so-called second spectrum $S_{2}\left(f_{2}, f\right)$, which is the power spectrum of the fluctuations of $S_{R}(f)$ with time [16]. $S_{2}\left(f_{2}, f\right)$ provides a direct probe of correlations between fluctuators: it is white (independent of $f_{2}$ ) for uncorrelated, and $S_{2} \propto 1 / f_{2}^{1-\beta}$ for interacting fluctuators [16]. At $B=0$, the glass transition in Si MOSFETs was manifested by a sudden and dramatic increase of $S_{R}$, a rapid rise of $\alpha$ from $\approx 1$ to $\approx 1.8[2,3]$, and a change of the exponent $(1-\beta)$ from a white (zero) to a nonwhite (nonzero) value 3 ]. We adopt similar criteria for the glass transition in $B$.

Figures 2(a), 2(b), and 2(c) present the $B$-field dependences of $S_{R}, \alpha$, and $(1-\beta)$, respectively, for several $n_{s}$. For each $n_{s}$ and $B, S_{2}\left(f_{2}, f\right)$ was calculated [17] for three different octaves $f: 2-4 \mathrm{mHz}, 4-8 \mathrm{mHz}$, and 8-16 $\mathrm{mHz}$. In order to reduce the uncertainty in $(1-\beta)$, the expo-



FIG. 2: (a) $S_{R}(f=1 \mathrm{mHz})$, (b) $\alpha$, and (c) $(1-\beta)$ vs. $B$ for $n_{s}\left(10^{10} \mathrm{~cm}^{-2}\right)$ shown on the plots; $T=0.24 \mathrm{~K}$. Other data have been omitted for clarity. $S_{R}(f)$ has been corrected for the white background noise. The arrow in (a) shows $B_{g}$ for $n_{s}\left(10^{10} \mathrm{~cm}^{-2}\right)=11.9$. The error bars on the right show maximum standard deviations of the data, which, for clarity, were plotted after performing a simple 3 -point average. The origin of the fluctuations of $S_{R}(B)$ for some $n_{s}$ at $B \gtrsim B_{g}(e$. $g$. for $\left.n_{s}\left(10^{10} \mathrm{~cm}^{-2}\right)=11.2\right)$ is not understood at this time.

nent shown in Fig. 2(c) represents the average $(1-\beta)$ obtained from $S_{2}$ in those three octaves.

At the highest $n_{s}$, in the metallic phase, "pure" $1 / f$ noise $(\alpha \simeq 1)$ resulting from uncorrelated $[(1-\beta) \approx 0]$ fluctuators is observed in $B=0[2,3]$. A decreasing $S_{R}$ with $B$ in Fig. 2(a) shows that parallel $B$ suppresses such noise, the behavior that is already apparent from the raw data [e.g. bottom two traces in Fig. 1(a)]. In addition, the exponent $\alpha$ is reduced to $\sim 0.5$ [Fig. 2(b)]. The dependence of both $S_{R}$ and $\alpha$ on $B$ becomes weaker for $B \gtrsim 3-4 \mathrm{~T}$. We note that noise in the metallic phase does not depend on $n_{s}$, similar to the $B=0$ case [2, 3]. Even though the origin of this noise is not known at the moment, these data should provide a valuable contribution towards understanding the nature of the metallic phase. Pure $1 / f$ noise in $B=0$ has been observed recently also in a 2D hole system in GaAs near the apparent MIT, on the metallic side [18]. 


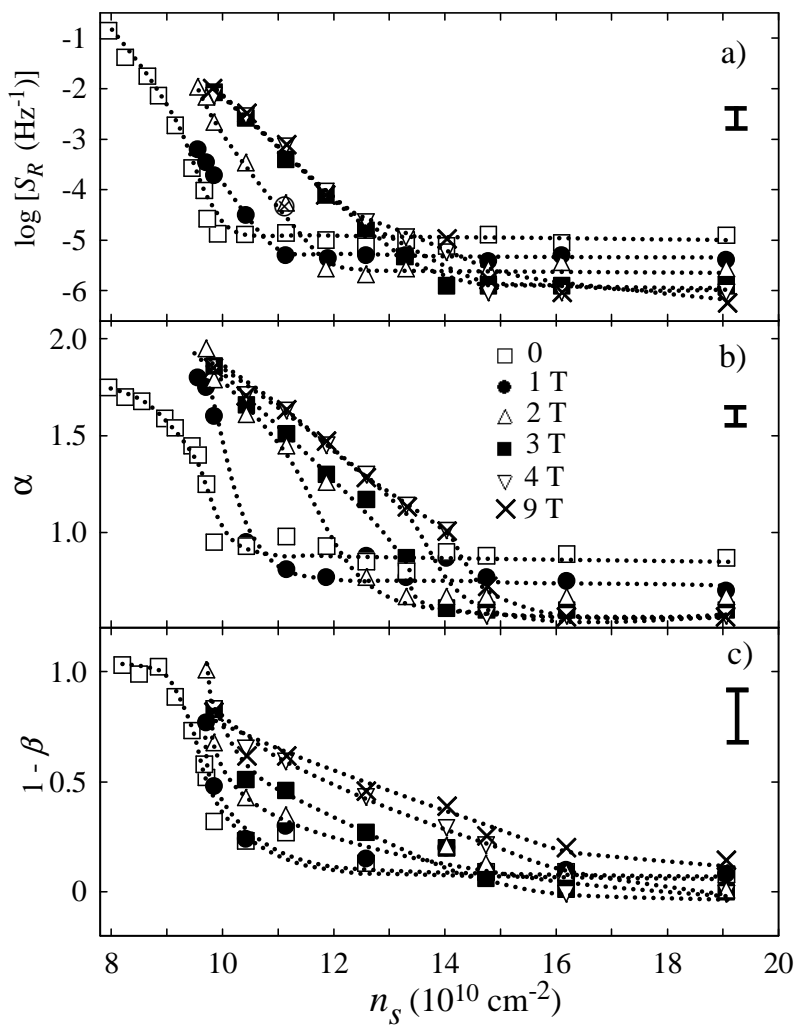

FIG. 3: (a) $S_{R}(f=1 \mathrm{mHz})$, (b) $\alpha$, and (c) $(1-\beta)$ vs. $n_{s}$ for several $B$ shown on the plot. The same symbols are used in all three panels. Dotted lines guide the eye. The maximum error bars are shown in the upper right.

At lower $n_{s}$, the noise at low $B$ behaves as described above. However, for each $n_{s}$, there is now a well-defined field $B_{g}$ [Fig. 2(a)] where, after the initial decrease with $B, S_{R}$ begins to increase dramatically, accompanied by a rapid rise of $\alpha$ and an increase of $(1-\beta)$ to nonwhite values, indicative of the onset of strong correlations. This striking change of both the magnitude and character of noise within a narrow range of fields is obvious even from the raw data (e.g. middle two traces in Fig. 1). In analogy with the $B=0$ case, we identify $B_{g}$ as the field where glass transition occurs at a given $n_{s}$. At even higher fields $(B \gtrsim 4 \mathrm{~T}), S_{R}, \alpha$, and $(1-\beta)$ no longer depend on $B$, consistent with the fact that the electrons are spin polarized. Nevertheless, Fig. 2 shows that the strong dependence of noise on $n_{s}$ is still present for a given $B$ in this regime. The dependence of $S_{R}, \alpha$, and $(1-\beta)$ on $n_{s}$ is plotted explicitly in Fig. 3 for several $B$. The density where $S_{R}$ begins to increase dramatically, accompanied by changes in $\alpha$ and $(1-\beta)$, is identified as the glass transition density $n_{g}$ for a given $B$.

The values of $B_{g}\left(n_{s}\right)$ and $n_{g}(B)$ determined in this way have been used to construct a phase diagram in the $\left(n_{s}, B\right)$ plane, as shown in Fig. 4 The square symbols designate the boundary of the glassy phase, and clearly show an increase of $n_{g}$ with $B$, followed by a saturation



FIG. 4: $T=0$ phase diagram. The dashed lines guide the eye. Squares: boundary between the metallic and MG phases; other symbols: boundary between the MG and insulating glass phases. The data from Ref. [13] have been shifted up by $0.85 \times 10^{10} \mathrm{~cm}^{-2}$ to make the $n_{c}(B=0)$ values coincide.

at higher fields. It is interesting to compare the behavior of $n_{g}(B)$ with $n_{c}(B)$. In Ref. [13], where samples almost identical to ours were used, $n_{c}(B)$ was determined based on both a vanishing activation energy and a vanishing nonlinearity of current-voltage characteristics when extrapolated from the insulating phase. We have used the activation energy method but the range of accessible $T$ and $n_{s}$ in our ac measurements was smaller compared to the dc technique and dilution refrigerator $T$ (down to $30 \mathrm{mK}$ ) used in Ref. [13]. We find that the data that we have available in the insulating regime are best described by $\langle\sigma\rangle \propto \exp \left[-\left(T_{0} / T\right)^{n}\right]$ with $n=1 / 2$, which corresponds to variable-range hopping with a Coulomb gap [19]. The extrapolation of $T_{0}\left(n_{s}\right)$ to zero, where only $n_{s}$ with $T_{0} \gtrsim 0.5 \mathrm{~K}$ were used, was employed to determine $n_{c}$ shown in Fig. 4. Strictly speaking, the rather limited range of data does not allow one to make an accurate distinction between different forms of activated $\langle\sigma(T)\rangle$. This experimental uncertainty is reflected in rather large error bars for $n_{c}$ shown in Fig. 4 Nevertheless, the agreement between our results and those obtained in Ref. 13] is remarkably good (Fig. 44).

Furthermore, it was possible for the first time to determine $n_{c}$ in a magnetic field by studying $\langle\sigma(T)\rangle$ on the metallic side of the MIT. In particular, in a relatively narrow range of $n_{s}$ for $B=1,2,3 \mathrm{~T}$, the data are best described by the metallic power-law behavior $\left\langle\sigma\left(n_{s}, B, T\right)\right\rangle=\left\langle\sigma\left(n_{s}, B, T=0\right)\right\rangle+b\left(n_{s}, B\right) T^{1.5}[$ Fig. [5(a)], similar to what was observed in the MG phase of highly disordered samples at $B=0$ [2]. The extrapolated $T=0$ conductivities go to zero at $n_{c}(B)$ in a power-law fashion $\left\langle\sigma\left(n_{s}, B, T=0\right)\right\rangle \propto \delta_{n}^{\mu}$ with $\mu \sim 1.5$ [see Fig. [5(b); $\left.\delta_{n}=n_{s} / n_{c}(B)-1\right]$, in agreement with theoretical expectations near a QPT 20]. At $B=0$, there is some evidence 21] of similar behavior with $\mu \sim 1-1.5$, ob- 

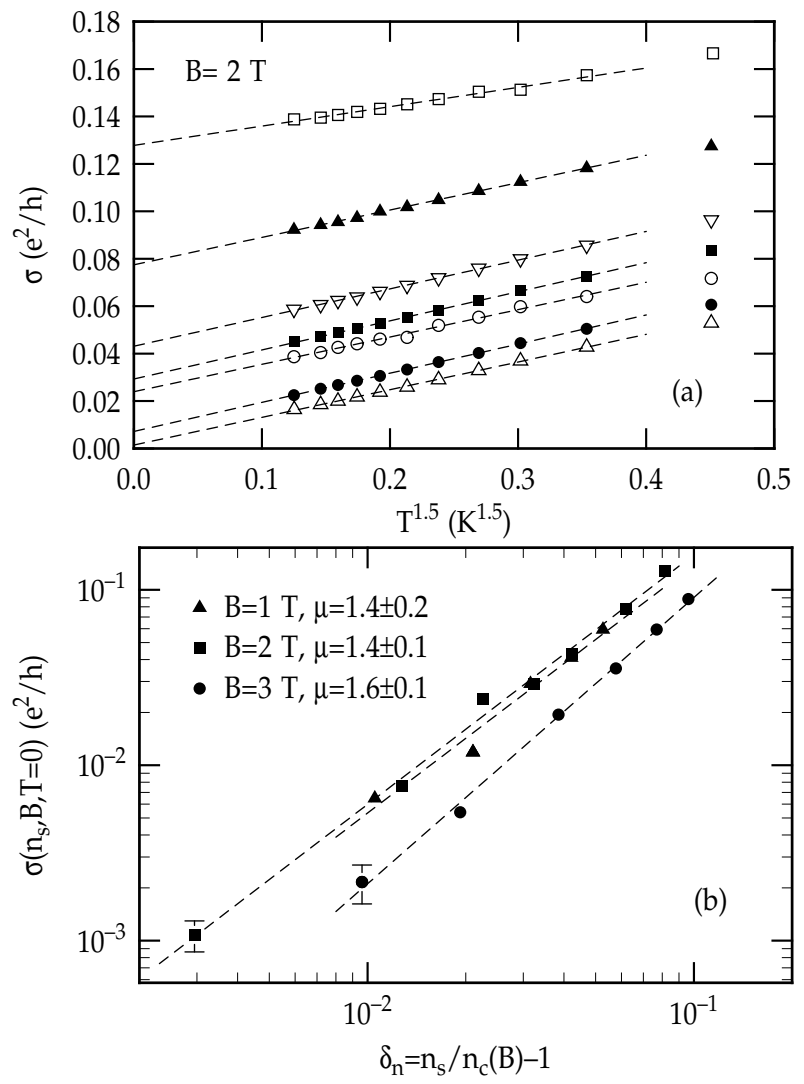

FIG. 5: (a) $\langle\sigma(T)\rangle$ in the MG phase for $n_{s}\left(10^{10} \mathrm{~cm}^{-2}\right)=$ 11.9, 11.6, 11.3, 11.2, 11.0, 10.9, 10.7 from top; $B=2 \mathrm{~T}$; $n_{c}(B=2 \mathrm{~T})=10.67 \times 10^{10} \mathrm{~cm}^{-2}$. (b) $\langle\sigma(T=0)\rangle \propto \delta_{n}^{\mu}$.

tained from $\sigma(T=0)$ in the metallic phase of different Si MOSFETs. Considering that $n_{c}(B)$ for $B=1,2,3 \mathrm{~T}$ shown in Fig. 4 have been obtained by approaching the MIT from the metallic side, the agreement between our results and those of Ref. 13] is even more remarkable.

The phase diagram in Fig. 4 makes it clear why the metallic $T^{3 / 2}$ temperature dependence of conductivity [Fig. 5(a)] is observed in such a narrow range of $n_{s}$ : it is characteristic of the metallic glass phase, even in $B$ of up to $3 \mathrm{~T}$. At $B=0$, the $\mathrm{MG}$ phase is very narrow and the $T^{3 / 2}$ correction is, therefore, difficult to observe in these samples, in contrast to highly disordered ones [2]. A parallel $B$ increases the width of the MG phase (Fig. 4). In addition, the range of $T$ where $T^{3 / 2}$ holds increases with parallel $B$ (not shown). The increase of $n_{g}$ and $n_{c}$, and the broadening of the MG phase with $B$ can be understood to result from the suppression of screening by a parallel $B$ 222], which increases the effective disorder. This, in turn, favors glassiness, consistent with theoretical expectations [9]. It is also interesting to note that the prefactor $b\left(n_{s}, B\right)$ of the $T^{3 / 2}$ correction [slopes in Fig. [5(a)] does not seem to depend on $n_{s}$, in contrast to the strong $n_{s}$-dependence seen in highly disordered (low-mobility) samples at $B=0$ [2]. Further careful in- vestigation is required in order to determine whether this difference can be attributed to the effects of $B$ or to the effects of disorder. Such a study, however, along with a detailed analysis of $\langle\sigma(T)\rangle$ in the MG phase at high fields, is beyond the scope of this paper.

In summary, the glass transition in high-mobility $\mathrm{Si}$ MOSFETs has been found to persist in high parallel $B$, where the 2DES is spin polarized. These results demonstrate that charge, as opposed to spin, degrees of freedom are responsible for glassy ordering of the 2DES near the MIT, consistent with recent theory [5, 8, 9].

We are grateful to V. Dobrosavljević for useful discussions. This work was supported by NSF grant No. DMR-0071668, and NHMFL through NSF Cooperative Agreement No. DMR-0084173.

* Also at Institute of Physics, PAS, Warsaw, Poland; Electronic address: jaroszy@magnet.fsu.edu

[1] E. Abrahams, S. V. Kravchenko, and M. P. Sarachik, Rev. Mod. Phys. 73, 251 (2000), and references therein.

[2] S. Bogdanovich and D. Popović, Phys. Rev. Lett. 88, 236401 (2002).

[3] J. Jaroszyński, D. Popović, and T. M. Klapwijk, Phys. Rev. Lett. 89, 276401 (2002).

[4] J. S. Thakur and D. Neilson, Phys. Rev. B 547674 (1996); 59, R5280 (1999).

[5] A. A. Pastor and V. Dobrosavljević, Phys. Rev. Lett. 83, 4642 (1999).

[6] S. Chakravarty et al., Philos. Mag. B 79, 859 (1999).

[7] S. Sachdev, Pramana 58, 285 (2002), cond-mat/0109309 Philos. Trans. R. Soc. London A 356, 173 (1998).

[8] D. Dalidovich et al., Phys. Rev. B 66, 081107 (2002).

[9] V. Dobrosavljević, D. Tanasković, and A. A. Pastor, Phys. Rev. Lett. 90, 016402 (2003).

[10] K. Binder et al., Rev. Mod. Phys. 58, 801 (1986).

[11] As usual, parallel field is used in order to avoid complications due to the orbital motion of electrons.

[12] V. M. Pudalov et al., Phys. Rev. Lett. 70, 1866 (1993).

[13] A. A. Shashkin et al., Phys. Rev. Lett. 87, 266402 (2001).

[14] R. Heemskerk Ph.D. thesis, University of Groningen, The Netherlands, 1998 (unpublished); R. Heemskerk and T. M. Klapwijk, Phys. Rev. B 58, R1754 (1998).

[15] T. Okamoto et al., Phys. Rev. Lett. 82, 3875 (1999); S. A. Vitkalov et al., Phys. Rev. Lett. 85, 2164 (2000); E. Tutuc et al., Phys. Rev. Lett. 86, 2858 (2001).

[16] M. B. Weissman, Rev. Mod. Phys. 60, 537 (1988); Rev. Mod. Phys. bf 65, 829 (1993); M. B. Weissman et al., J. Magn. Magn. Mater. 114, 87 (1992), and references therein.

[17] G. T. Seidler and S.A. Solin, Phys. Rev. B 53, 9753 (1996); K. M. Abkemeier, Phys. Rev. B 55, 7005 (1997).

[18] R. Leturcq et al., Phys. Rev. Lett. 90, 076402 (2003).

[19] A. L. Efros and B. I. Shklovskii, J. Phys. C 8, L49 (1975).

[20] D. Belitz et al., Rev. Mod. Phys. 66, 261 (1994).

[21] R. Fletcher et al., Semicond. Sci. Tech. 16, 386 (2001).

[22] V. T. Dolgopolov and A. Gold, JETP Lett. 71, 27 (2000); I. F. Herbut, Phys. Rev. B 63, 113102 (2001). 